\title{
Aktueller Forschungsstand und Status Quo
}

\subsection{Ergebnisse aktueller Studien}

Die kognitiven Fähigkeiten der Kinder werden durch frühe Bildungsmaßnahmen positiv beeinflusst (Anger et al., 2017, 16 f.; Autorengruppe Bildungsberichterstattung, 2018, 74). Diese umfassen sprachliche und mathematische Kompetenzen ebenso wie das Problemlösen. Förderliche Effekte konnten insbesondere auf sozial schwächer gestellte Kinder und Kinder mit Migrationshintergrund nachgewiesen werden, wobei diese deutlich seltener Tageseinrichtungen besuchen (Anders, 2013). Die Chance kognitiver Förderung während der frühen Lebensjahre ist größer als in späterem Alter und hat einen positiven Einfluss auf die nachfolgenden Entwicklungsschritte (Camehl, 2016). Die nicht-kognitiven Fähigkeiten beziehen sich auf die sozialen und emotionalen Kompetenzen der Kinder und schließen auch die Persönlichkeitsmerkmale mit ein. Sie fördern den Erwerb der kognitiven Fähigkeiten und korrelieren ebenfalls positiv mit dem späteren Erfolg auf dem Arbeitsmarkt (Almlund et al., 2011, 152-156). In einer Übersichtsarbeit konnte Camehl (2016) für die in Deutschland durchgeführten Studien positive Effekte zwischen nicht-kognitiven Fähigkeiten und Besuch einer Kindertageseinrichtung aufzeigen, insbesondere positiv waren die Effekte für Kinder mit niedrigem sozioökonomischem Hintergrund. Im Unterschied dazu stellten sich die Effekte von internationalen Studien uneindeutig dar. Es konnten ebenso positive wie negative als auch keine Effekte nachgewiesen werden (Camehl, 2016); dies aufgrund des unterschiedlichen Eintrittsalters der Kinder in die Betreuung, des Alters bei der Ergebnismessung und der Qualitätsunterschiede der Einrichtungen. Bach et al. (2018) konnten einen positiven Effekt früher Fremdbetreuung ab drei Jahren auf Jugendliche in Bezug auf Kommunikations- und Durchsetzungsfähigkeit belegen, während Kühnle und Oberfichtner (2017) keine langfristigen 
Effekte feststellten, weder für Kinder mit niedrigem sozioökonomischem noch mit Migrationshintergrund. Camehl (2016) postuliert, dass qualitative Einflussgrößen der Betreuung einen förderlichen Effekt auf die Kompetenzen der Kinder haben, insbesondere auf das prosoziale Verhalten bei vier- bis fünfjährigen Kindern (Camehl \& Peter, 2017). Dieser Effekt war bei in kleinen Gruppen betreuten Kindern mit Müttern niedrigen Bildungsstands am größten.

Die Prozessqualität pädagogischer Pflege und Betreuung von zwei bis vierjährigen Kindern in Einrichtungen der Tagespflege fällt laut den Ergebnissen der NUBBEK-Studie insgesamt unbefriedigend aus (Tietze, Becker-Stoll, Bensel, Eckhardt, Haug-Schnabel, Kalicki, Keller \& Leyendecker, 2013, 2012) und kann nicht allein durch verbesserte Rahmenbedingungen, wie eine verbesserte Fachkraft-Kind-Relation, behoben werden. Da der Entwicklungsstand der Kinder mehr von familiären Faktoren als von externen Betreuungsfaktoren abhängt, wobei Kinder mit Migrationshintergrund besonders negativ betroffen sind, empfehlen die Autoren eine stärkere Zusammenarbeit mit den Familien.

Die Ausbildung von Erziehern für den U3-Bereich ist bislang unzureichend, zudem scheitert laut einer bundesweiten Fragebogenstudie (Jungbauer \& Biedenbach, 2016) der Wissenstransfer in die Praxis aufgrund struktureller und individueller Umstände. Die Arbeit mit unter Dreijährigen wurde von den Erziehern anstrengender bewertet als die mit älteren Kindern. Die offiziellen Anforderungen an das Fachpersonal decken sich nur unzureichend mit der subjektiven Einstellung der Erzieher bezüglich der professionellen Haltung und weisen laut der Autoren Diskrepanzen auf. Während die Bewertung der eigenen Arbeit vielfach positiv ausfiel, hielt die Hälfte der Befragten eine U3-Betreuung eigener Kinder für bedenklich, sodass Jungbauer et al. (2016) Struktur- und Prozessmängel hinsichtlich des Personalschlüssels, Gruppengröße und Ausbildung konstatieren. Diese könnten durch weitere Investitionen in den U3-Bereich und Anheben des Fachkräfte-Status anhand einer höheren Vergütung erreicht werden.

Die Wiener Krippenstudie untersuchte das Erleben von Kindern zwischen 10 und 33 Monaten vor, während und nach der Eingewöhnung in die Fremdbetreuung (Ahnert, Kappler \& Eckstein-Madry, 2012; Datler, Ereky-Stevens, Hoover-Reisner \& Malmberg, 2012). Die Forscher konnten eine erhöhte Stressbelastung anhand des Cortisolspiegels der Kinder nachweisen, insbesondere in Zusammenhang mit der Dauer der Eingewöhnungszeit, die bei allen Kindern höchst individuell, und teilweise über Monate hinweg, verlief. Das kindliche sowie mütterliche Temperament, die Bindungsqualität und Copingstrategien zur Stressbewältigung waren ebenfalls von Bedeutung (Ahnert et al., 2012; Datler et al., 2012). Insbesondere stille Kinder können durch eine frühe Fremdbetreuung belastet sein, was häufig vom Betreuungspersonal als Anpassung interpretiert 
werde. Ausreichend mit den Eltern verbrachte Zeit zur Affektregulation ist für die Kinder wichtig.

In einer Meta-Studie untersuchten Vermeer und van Ijzendoorn (2006) europäische und amerikanische Daten und konnten belegen, dass in der Fremdbetreuung die Cortisolwerte der Kinder höher waren als im häuslichen Setting. Zudem zeigte sich, mit einem über den Tag signifikant ansteigenden Cortisolspiegel, ein abnormer Verlauf. Als bedeutsamsten Moderator konnten die Forscher das Alter der Kinder von unter 36 Monaten identifizieren (Vermeer \& van Ijzendoorn, 2006, 397; Vermeer \& Groeneveld, 2017). Sie führen ihre Ergebnisse auf eine unzureichende Stressregulation durch anstrengend erlebte Interaktionen zurück.

Unter dem Konflikt, Beruf und Familie miteinander zu vereinbaren, leiden Mütter und Väter gleichermaßen, wobei Frauen sich durch familiäre Pflichten in ihrer beruflichen Entwicklung gehemmt sehen (Shockley, Shen, DeNunzio, Arvan \& Knudnsen, 2017). Für die Vereinbarung von Familie und Beruf ist ein flexibles Angebot an Betreuungseinrichtungen erforderlich, dass sich an den Arbeitszeiten orientiert (BMFSFJ, 2016, 14). Aktuell fehlen etwa 300000 Plätze in der U3-Betreuung (Geis, 2018). Zugleich wird ein weiter zunehmender Engpass von Fachkräften prognostiziert (Autorengruppe Fachkräftebarometer, 2017, 177-185) und ein Personalnotstand nicht ausgeschlossen (Rauschenbach, Schilling \& Meiner-Teubner, 2017).

In den beiden folgenden Abschnitten wird der Status quo der institutionellen Fremdbetreuung und der Erwerbstätigkeit von Müttern anhand von Zahlen, Daten und Fakten belegt, um die Untersuchungsbasis abzuschließen.

\subsection{Institutionelle Fremdbetreuung von Kleinkindern}

Mit diesem Abschnitt werden die ordnungspolitischen Rahmenbedingungen zu Tagesstättenausbau und Förderungsaspekt sowie die Betreuungsquoten im Zeitverlauf, Betreuungsumfang und Elternwünsche im Ost-West-Vergleich vorgestellt.

Die Rahmengesetzgebung des Bundes wird auf Landesebene umgesetzt. Neben dem SGB IIIV, das in Abschnitt 2.3.1 bereits erwähnt wurde, ist mit dem Gesetz zum qualitätsorientierten und bedarfsgerechten Ausbau der Tagesbetreuung für Kinder (Tagesbetreuungsausbaugesetz - TAG) im Jahr 2004 und dem Gesetz zur Förderung von Kindern unter drei Jahren in Tageseinrichtungen und Kindertagespflege (Kinderförderungsgesetz - KiföG) im Jahr 2008 ein quantitativer und qualitativer Ausbau der Tagesbetreuung beschlossen worden. Seit dem Jahr 2013 ist ein Anspruch auf einen Betreuungsplatz ab dem vollendeten ersten Lebensjahr gesetzlich verankert. Aufgabe der Tagespflege ist die soziale, 
emotionale, körperliche und geistige Entwicklungsförderung, orientiert an den kindlichen Bedürfnissen und deren Wohl, die Vermittlung von Werten, Unterstützung und Ergänzung der Familien bei Erziehung und Bildung sowie Hilfe zur besseren Vereinbarung von Erwerbstätigkeit und Kindererziehung ( $22,22 a$, 23, 24 SGB IIIV). Die Betreuungsaufgabe wurde vom Aspekt der frühkindlichen Bildung als Leitmotiv abgelöst und hat damit auch das Berufsbild und die Anforderungen an die Erzieher verändert (Rudolph, 2012, 15). Seit dem Jahr 2006 hat sich die Zahl der betreuten Kinder unter drei Jahren deutlich erhöht, das entspricht einem Anstieg der Quote im Bundesdurchschnitt von 13,6 \% im Jahr 2006 (BMFSFJ, 2017) auf 33,6 \% im Jahr 2018 (Statistisches Bundesamt, 2018). In den neuen Bundesländern betrug die Quote $51,5 \%$, in den westdeutschen dagegen $29,4 \%$. Die unterschiedliche Inanspruchnahme zeigt sich auch im detaillierten Altersvergleich. Während die Untereinjährigen in den westdeutschen Ländern nur zu 1,8 \%, die Einjährigen zu 29,5\% und die Zweijährigen zu 57,5\% in Einrichtungen betreut wurden, waren es in des neuen Ländern 3,1\% der unter Einjährigen, 65,2 \% der Einjährigen und 85,4\% der zweijährigen Kinder. Die folgende Abbildung zeigt den Anstieg der Betreuungsquoten von Kindern unter drei Jahren in der Tagesbetreuung über den Zeitraum von 2006 bis 2017, getrennt nach ost- und westdeutschen Bundesländern (Abbildung 3.1).

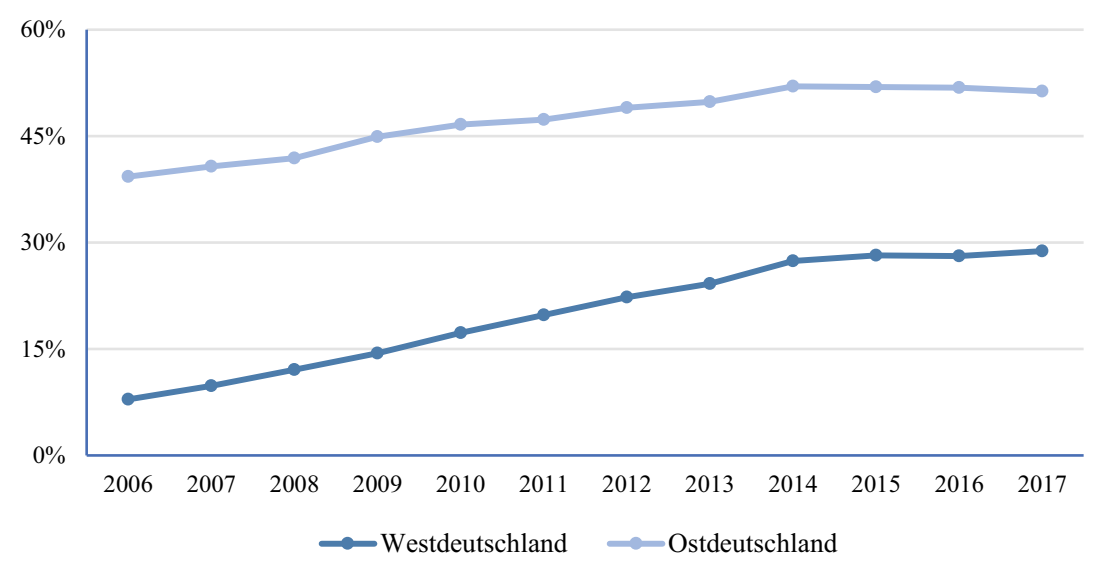

Abb. 3.1 U3-Betreuungsquoten von 2006 bis 2017 in Ost- und Westdeutschland. (Quelle: eigene Darstellung in Anlehnung an BMFSFJ, 2017, S. 9) 
Um den Betreuungsbedarf der Eltern von 42,5\% zu decken und die Differenz zur Betreuungsquote von 12,1\% auszugleichen, werden bis zum Jahr 2025 weitere 300700 Plätze für unter Dreijährige benötigt (BMFSFJ, 2017, 14). Rund 630000 Fachkräfte deckten den Bedarf im Jahr 2017 ab (Statistisches Bundesamt, 2018b) und betreuten insgesamt 762361 Kinder unter drei Jahren. Die von den Eltern nachgefragten Betreuungsumfänge stellten sich im Jahr 2017 sehr unterschiedlich dar. Einen Halbtagsplatz mit maximal 25 Wochenstunden wünschten $17 \%$ und 25 bis 35 Wochenstunden $27 \%$ der Eltern. Ein Ganztagsplatz mit bis zu 45 Stunden pro Woche entsprach zu $34 \%$ und mehr als 45 Wochenstunden zu $20 \%$ dem Elternwunsch (Alt, Gesell, Hubert, Hüsken, Kuhnke \& Lippert, 2017, 20), wobei insgesamt $76 \%$ der Eltern aus den neuen Bundesländern eine ganztägige Betreuung präferierten. In den alten Bundesländern war dies nur zu $46 \%$ der Elternwunsch (Alt et al., 2017, 21). Die tatsächliche U3-Ganztagsquote im Jahr 2017 betrug im Osten 39,9 \% und im Westen 13,4 \% (Wirtschafts- und Sozialwissenschaftliches Institut der Hans-Böckler-Stiftung - WSI, 2018). Nicht nur Betreuungsbedarf, -umfang und -quoten sind im Osten höher, auch halten die Tageseinrichtungen längere Öffnungszeiten vor als im Westen (BMFSFJ, 2017, 5). Mit Inkrafttreten des Gesetzes zur Weiterentwicklung der Qualität und zur Teilhabe in der Kindertagesbetreuung (KiQuTG) in diesem Jahr wurden zehn Handlungsfelder zur Verbesserung der Qualität in Kitas beschlossen. Unter anderem waren dies die Gewinnung und Sicherung qualifizierter Fachkräfte, ein guter Fachkraft-Kind-Schlüssel und bedarfsgerechte Öffnungszeiten (KiQuTG, 2018). Die Kosten für einen Betreuungsplatz unterscheiden sich regional stark und sind abhängig von Betreuungsumfang, Alter des Kindes und dem Einkommen der Eltern. Sie reichen von mehreren hundert Euro pro Monat bis hin zur Beitragsfreiheit (Geis-Thöne, 2018a). Die Betreuungsmaßnahmen sollen Müttern eine Berufsausübung erleichtern, welche im Folgenden näher beleuchtet wird.

\subsection{Erwerbsarbeit von Müttern}

In diesem Abschnitt soll sich die Vorstellung der ordnungspolitischen Rahmenbedingungen auf eine in Bezug zur Themenstellung relevante unterhaltsrechtliche Veränderung beschränken, die das Erwerbsverhalten von Müttern beeinflusst. Eine umfängliche Betrachtung der staatlichen Familienleistungen, wie zum Beispiel Kindergeld und -zuschlag oder Leistungen zu Bildung und Teilhabe, bleibt außen vor. Diese sind zur Beantwortung der Fragestellung nicht erforderlich, weil sie das Erwerbsverhalten von Müttern nur am Rande beeinflussen. Der Fokus liegt hingegen auf Beschäftigungsquote und -umfang, Verdienst und unbezahlter 
Arbeit im Vergleich zu Kinderlosen und Vätern sowie den Auswirkungen auf die Alterssicherung im Ost-West-Vergleich.

Die Erwerbsquote von Frauen ist seit dem Jahr 2007 von 69,2 \% auf 74,0 \% im Jahr 2017 gestiegen (BA, 2019, 6), diese Tendenz zur Erwerbsneigung wurde auch durch den Ausbau der Ganztagsbetreuung befördert (Büchel \& Spieß, 2002, 95). Die Beschäftigungsquote der Männer lag deutschlandweit im Jahr 2018 bei 63,3 \%, die der Frauen bei 56,5\%, wobei sich die Quote bei den ostdeutschen Frauen mit 60,8 \% von den westdeutschen Frauen mit 55,5\% unterschied (BA, 2019, 16). Teilzeit erwerbstätig waren $48 \%$ der Frauen, was nur zu $11 \%$ auf die Männer zutraf (BA, 2019, 14). Als Grund nannten die Frauen zu 28,5 \% Pflege und die Betreuung von Kindern, die Männer gaben dies nur zu 3,7 \% an (Statistisches Bundesamt - Destatis, 2018a, 157). Der Brutto-Verdienst von Frauen lag $13 \%$ unterhalb dem der Männer, in Führungspositionen, in denen Frauen zu etwa $26 \%$ repräsentiert sind, fiel die Vergütung durchschnittlich $27 \%$ geringer aus (BA, 2019, 13 f.). Die bereinigte Verdienstlücke betrug im Jahr 2014 rund $6 \%$ (Destatis, 2017), sodass sich durch niedrigere Erwerbsbeteiligung, Teilzeitarbeit, familienbedingte Erwerbspausen und die Arbeit in weniger gut vergüteten Berufen (Grabka, Jotzo, Rasner \& Westermeier, 2017) eine Rentenlücke von $42 \%$ im Westen und $23 \%$ im Osten zwischen Männern und Frauen ergab. Wrohlich und Zucco (2017) konnten die Verdienstlücke jedoch auch aus geschlechtsspezifischen Vergütungsunterschieden innerhalb einzelner Berufssparten erklären.

Die Erwerbstätigenquote von Eltern unterscheidet sich im Vergleich zu Kinderlosen, auch in Abhängigkeit von Kinderzahl und -alter. Die Erwerbsquote kinderloser Frauen lag im Jahr 2017 mit 67,4 \% nur knapp unterhalb der kinderloser Männer mit 72,2 \% (WSI, 2018). Während Väter, unabhängig vom Alter der Kinder, ihre Erwerbsquote auf 80,0 \% bis 84,2\% ausweiteten, war die Quote von Müttern durch das Alter der Kinder beeinflusst. Sie reduzierte sich auf 32,3\% mit Kindern unter drei Jahren und weitete sich mit dem Älterwerden der Kinder wieder aus: mit drei- bis fünfjährigen Kindern auf 63,6 \%, mit Sechs- bis Neunjährigen auf 69,9\% und mit Zehn- bis Vierzehnjährigen auf 74,1\%. Damit lag die Erwerbsquote von Müttern zehn- bis vierzehnjähriger Kinder über der kinderloser Frauen (WSI, 2018). Die Wochenarbeitszeit der Mütter verringerte sich mit der Anzahl der Kinder ebenfalls, was bei den Vätern nicht der Fall war. Im Jahr 2015 betrug die wöchentliche Arbeitszeit von Frauen im Bundesdurchschnitt 32,1 Stunden. Mütter im Westen mit einem Kind arbeiteten 26,4 Stunden, mit zwei Kindern 22,5 Stunden und mit drei und mehr Kindern 19,9 Stunden pro Woche. Im Vergleich dazu arbeiteten die Mütter im Osten mit Kindern im Durchschnitt 8,5 Stunden wöchentlich länger (WSI, 2016) und zeigen damit ein anderes Erwerbsverhalten als Mütter in Westdeutschland (Grabka et al., 2017). 
Mütter leisten zwar weniger Erwerbsarbeit als Väter, dafür ist ihr Anteil an unbezahlter Arbeit durch Kinderbetreuung und Hausarbeit größer, insbesondere dann, wenn Kinder unter sechs Jahren zu betreuen sind (Samtleben, 2019). Mütter wenden an Wochentagen insgesamt 13,5 Stunden für Erwerbsarbeit, Kinderbetreuung und Haushaltsorganisation auf, die Väter hingegen 12,5 Stunden. An Sonntagen wenden Väter 8,5 Stunden auf, die Mütter sind rund zwölf Stunden beschäftigt. Im Vergleich dazu wenden kinderlose Paare an Wochentagen jeweils rund zehn Stunden auf, mit einem Mehr an Erwerbsarbeit der Männer und einem Mehr an Haushaltstätigkeiten der Frauen. An Sonntagen beträgt die Zeitverwendung von Frauen insgesamt knapp drei Stunden, wobei sie eine halbe Stunde mehr Zeit für den Haushalt aufwenden als Männer, die insgesamt gut zwei Stunden Zeit investieren (Samtleben, 2019). Zusammenfassend lässt sich festhalten, dass Mütter mit Kindern unter sechs Jahren an Wochentagen 1,5 und an Sonntagen 3 Stunden mehr Zeit aufwenden als Väter. Im Vergleich zu Kinderlosen haben Mütter wochentäglich durchschnittlich 3 Stunden und an Sonntagen 9,5 Stunden weniger Zeit zur freien Verfügung (Samtleben, 2019). Die steigende Erwerbsbeteiligung von Frauen während der letzten zwei Jahrzehnte führte zu einer steten Reduzierung ihrer mit Kinderbetreuung und Haushaltsorganisation verbrachten Zeit. Samtleben (2019) stellt fest, dass die geringe Mehrbeteiligung der Männer an diesen unbezahlten Tätigkeiten seitdem nur aus der zeitlichen Enge bei den Frauen resultiert und kein freiwilliges Engagement darstellt.

Der Bezug von Elterngeld, der als Lohnersatzleistung während der ersten 14 Lebensmonate des Kindes seit dem Jahr 2007 gezahlt wird, führte zu einer Reduktion der Erwerbsbeteiligung von Müttern während des ersten Lebensjahres des Kindes (BMFSFJ, 2014, 63). Sie entschieden sich im Jahr 2014 zu 89,3\% für die maximale Bezugsdauer von zwölf beziehungsweise vierzehn Monaten. Knapp $80 \%$ der Väter nahmen im Jahr 2014 nur die zur maximalen Bezugsdauer erforderlichen beiden Partnermonate wahr, obwohl sie einkommensbedingt deutlich höhere Geldleistungen erhalten als Mütter (Geis-Thöne, 2018b). Die Ausübung einer Teilzeittätigkeit wird seit dem Jahr 2015 durch das ElterngeldPlus unterstützt, das bis maximal zum 24. Lebensmonat des Kindes ausgezahlt wird, um den frühen Wiedereinstieg in den Beruf beider Elternteile zu ermöglichen (BMFSFJ, 2015).

Mit der Unterhaltsrechtsreform im Jahr 2008 wurde die nacheheliche Eigenverantwortung gestärkt und zum Grundsatz erklärt (Gesetz zur Änderung des Unterhaltsrechts, 2007). So hat jeder geschiedene Ehepartner spätestens ab dem dritten Lebensjahr eines zu betreuenden Kindes für den eigenen Lebensunterhalt aufzukommen, ein Unterhaltsanspruch ist damit häufig nicht mehr gegeben. 
Von dieser Gesetzesänderung sind insbesondere alleinerziehende Mütter betroffen. Im Jahr 2015 gab es ca. 1,6 Mio. Alleinerziehende, die rund 2,3 Mio. minderjährige Kinder betreuten; neun von zehn Alleinerziehenden sind Mütter (BMFSFJ, 2018). Obwohl $68 \%$ aktiv erwerbstätig sind, davon $42 \%$ in Vollzeit, sind $39 \%$ der alleinerziehenden Bedarfsgemeinschaften auf Grundsicherungsleistungen angewiesen und gelten als besonders armutsgefährdet (BMFSFJ, 2018).

Zusammenfassend lässt sich festhalten, dass Mütter in Abhängigkeit von Kinderzahl und-alter häufiger Erwerbsunterbrechungen haben und Teilzeit erwerbstätig sind, über die Lebensspanne weniger verdienen, mehr unbezahlte Arbeit leisten als Väter und Kinderlose und weniger Erholungszeit haben. Zudem sind sie häufiger alleinerziehend und generell mehr von Altersarmut betroffen als Männer, im Westen stärker als im Osten.

Nach Abschluss der Analysebasis, die in Form von verschiedenen theoretischen Ansätzen und aktuellen Daten vorgestellt wurde, erfolgt im nächsten Abschnitt die Erläuterung und Begründung des methodischen Vorgehens, bevor dann nachfolgend die Ergebnisse der Untersuchung vorgelegt werden.

Open Access Dieses Kapitel wird unter der Creative Commons Namensnennung 4.0 International Lizenz (http://creativecommons.org/licenses/by/4.0/deed.de) veröffentlicht, welche die Nutzung, Vervielfältigung, Bearbeitung, Verbreitung und Wiedergabe in jeglichem Medium und Format erlaubt, sofern Sie den/die ursprünglichen Autor(en) und die Quelle ordnungsgemäß nennen, einen Link zur Creative Commons Lizenz beifügen und angeben, ob Änderungen vorgenommen wurden.

Die in diesem Kapitel enthaltenen Bilder und sonstiges Drittmaterial unterliegen ebenfalls der genannten Creative Commons Lizenz, sofern sich aus der Abbildungslegende nichts anderes ergibt. Sofern das betreffende Material nicht unter der genannten Creative Commons Lizenz steht und die betreffende Handlung nicht nach gesetzlichen Vorschriften erlaubt ist, ist für die oben aufgeführten Weiterverwendungen des Materials die Einwilligung des jeweiligen Rechteinhabers einzuholen.

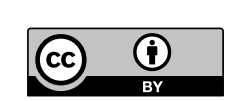

